\title{
Les figures hybrides dans Les Chemins qui montent de Mouloud Feraoun
}

\author{
Chia-hua Hsu \\ Université Nationale Centrale (Taiwan)
}

Les années cinquante voient une floraison de romans francophones d'écrivains algériens qui placent les questions identitaires et le rapport avec la tutelle coloniale au centre de leur préoccupation. Loin de reproduire la dichotomie en vogue entre la France-colonisateur et l'Algérie-colonisé, ces œuvres déploient, examinent et nuancent la pluralité d'identités et d'appartenances qui est au cœur des situations dans lesquelles se trouvent et les personnages et leurs créateurs. Le nombre de récits de formation, souvent à caractère autobiographique, atteste la préférence de ces auteurs pour cette forme narrative qui s'avère féconde pour mettre en lumière, à travers le parcours d'un enfant du pays qui s'arrache peu à peu à son milieu socioculturel d'origine, l'acculturation au système éducatif français ainsi que le sentiment ou la réalité de déracinement qui s'ensuit. Outre cette figure typique d'enfant acculturé à cheval entre deux systèmes discursifs, socioculturels et linguistiques, on identifie également de nombreuses figures hybrides ou métisses se trouvant à la frontière à mi-chemin ou simultanément entre deux voire plusieurs territorialités. Chacune porte en soi des caractères d'hybridité, qui sont les fruits du croisement des différentes cultures, langues et conventions sociohistoriques et qui créent un "tiers espace» depuis lequel s'articulent des formes alternatives d'identité selon la théorie avancée par Homi Bhabha (77-83) ${ }^{1}$. Ce qui intéresse les romanciers algériens de cette époque est moins l'hybridité en tant que résultat fini de la transculturation que les diverses manières dont les figures hybrides réagissent aux normes ou que le rapport et la tension entre ces figures de marginalité et l'autorité normative qui siège au centre.

L'œuvre de Mouloud Feraoun s'inscrit dans le sillage de cette préoccupation et explore la question de l'hybridité et le lieu interstitiel où

\footnotetext{
1 Selon Bhabha, l'hybridité est un «tiers espace », un espace « interstitiel» dont la logique n'est pas de remonter aux deux moments ou lieux originaires d'un hybride, mais d'affirmer l'hybridation comme un processus générateur de nouvelle identité qui est capable de défier les normes identitaires. Bhabha insiste sur «le caractère inachevé et transitoire des identités» (Simon 40) et sur le processus en cours dans cet espace de négociation perpétuelle à la frontière des identités fixes et essentialistes. Reprenant l'idée de Bhabha dans son étude sur l'hybridité culturelle, Simon souligne également que " [1]'hybride [...] n'est pas synonyme de fusion. L'hybride désigne un moment instable dans la vie des cultures, une situation de tension et d'inconfort face aux catégories existantes. » (Simon 32)
} 
elle habite à travers ses personnages et les situations mises en scène tout en ne négligeant rien des nuances possibles. Les diverses situations d'entre-deux et de croisement des deux territorialités à la fois qui abondent dans cette œuvre créent un lieu ou un mi-lieu fructueux pour l'exploitation de la question d'hybridité et de métissage dans ses sens multiples. Le Fils du pawvre (1950), premier roman de Feraoun et roman d'apprentissage, met en scène l'enfance et la formation à l'école française du petit Fouroulou Menrad dans les années 1910-1920, dont l'histoire est inspirée grandement de la vie personnelle de l'auteur, qui a connu le même parcours d'enfant kabyle devenu instituteur. Son deuxième roman, La Terre et le sang (1953) et le troisième roman Les Chemins qui montent (1957), que Robert Elbaz et Martine Mathieu-Job considèrent comme un «seul et même Roman » (45), narrent successivement l'histoire du retour en Kabylie d'un couple franco-kabyle après une période d'exil en France et celle du fils métis de ce même couple en proie à un dédoublement identitaire. S'il s'agit dans le premier roman de l'hybridation - le processus qui a pour résultat l'hybridité - qui se dessine à travers la formation d'un enfant ayant évolué à la rencontre de deux cultures et de deux discours, l'une d'origine (kabyle) et l'autre d'adoption (française), celle-ci va au-delà d'un mélange au niveau particulièrement socioculturel et touche également au métissage biologique dans les deux ouvrages qui suivent.

La présente étude se focalisera sur les figures hybrides et métisses dans Les Chemins qui montent, dans le but d'examiner les situations d'entre-deux et les multiples niveaux d'enjeux concernant la notion de l'hybridité et du métissage à la frontière où les deux forces se rencontrent ou dans un tiers espace où elles convergent. Nous nous intéresserons particulièrement à ce mi-lieu ou l'entre-lieu - lieu liminal entre deux frontières où l'hybridité et le métissage auront lieu - et aux négociations identitaires effectuées par les personnages dans cet espace problématique. Notre analyse portera sur les diverses manifestations de l'hybridité à travers l'étude des personnages afin de présenter un éventail de figures hybrides au cœur de l'œuvre féraounienne et qui constituent la société kabyle à l'apogée et vers la fin de la colonisation française.

\section{Amer, le sang-mêlé}

Tout commence par l'histoire de l'émigration des Kabyles vers la France et les allers-retours perpétuels des émigrés. Des déplacements transméditerranéens commencent vers le début du vingtième siècle et deviennent ensuite pour les Kabyles une trajectoire migratrice régulière. L'écrivain situe l'histoire du couple mixte de La Terre et le sang, le roman précédant Les Chemins qui montent et la première partie de sa «chronique allant de 1910-1950»(Feraoun, Lettres 54), dans ce contexte historique spécifique et installe dans le décor kabyle une Française prénommée Marie, compagne d'Amer-ou-Kaci et surnommée d'un ton révérencieux 
«Madame» (Feraoun, TS 36) par les villageois dès son arrivée au village. À la fin du récit, Amer est mort, et l'enfant que porte Marie présage l'arrivée d'un héritier qui assurerait le relais du sang et la place au village de la famille désormais sans mâle. Or, si l'intégration de Marie semble se dérouler dans des conditions idéales si bien qu'elle finit par "se kabyliser » (Feraoun, TS 99) dans La Terre et le sang, la reconnaissance de son fils métis comme un enfant du pays s'avère être une conquête ardue dans Les Chemins qui montent.

«Je m'appelle Amer n'Amer, autrement dit Amer fils d'Amer. Cela suffit pour me distinguer de tous les Amer du village parce que, chez nous, on n'est jamais quelqu'un fils du même quelqu'un. »(Feraoun, CM 103) Le protagoniste commence son journal en s'identifiant dès la première ligne et annonce d'emblée l'une des problématiques centrales du roman: comment se définir et se distinguer des autres, comment se reconnaittre et se faire reconnaitre par les autres, comment être soi au sein de sa propre communauté. Ce double prénom trace une ligne de démarcation entre l'individu, ses origines et la collectivité : il est à la fois l'affirmation de son individualité, de son appartenance et de son rapport avec le monde. Il n'est pas innocent pour de multiples raisons. Tout d'abord, ce prénom affirme l'origine paternelle d'Amer fils et son appartenance incontestable à la famille du père. Amer fils d'Amer assure la continuation de la lignée familiale et remédie à la disparition prématurée de son ascendant paternel. Dans cette perspective, il est l'héritier authentique de la famille kabyle du père, c'est un Amer en sa double qualité de père et de fils. Néanmoins, ce prénom lui confère en même temps une singularité voire même un caractère excentrique au sein d'une société où la notion de famille constitue le fondement autour duquel régit la communauté. Le réseau familial, autrement dit le réseau social basé sur le sang et sur l'alliance matrimoniale, définit les relations humaines dans la vie communautaire tant du village d'Ighil-Nezman que de la société kabyle en général ; aspect que Feraoun a maintes fois expliqué dans ses divers écrits, comme ses trois romans en témoignent. Or, cette dénomination inhabituelle met en relief la qualité d'orphelin sans père ni soutien de son détenteur, sans compter le fait de sa double origine :

«Moi, j’ai repris le prénom de mon père parce qu'il était mort, lui aussi, quand je suis né. [...] Ma mère est française. [...] Je suis fils unique. J'ai un double prénom, une mère qui ne ressemble pas aux autres et $j$ 'ai été orphelin dans le ventre de cette dernière. C'est assez singulier, tout cela. » (CM 103)

C'est une situation difficile à vivre dans une société où le lien de parenté l'emporte sur l'individualité et où la place d'un individu dépend de sa filiation. Par conséquent, le double prénom signale la présence et l'absence simultanées du père, et «Amer est et n'est pas son père » car il répète tout

${ }^{2}$ Nous emploierons désormais l'abréviation $C M$ sans donner le nom de l'auteur pour tout renvoi aux Chemins qui montent de Feraoun dans cet article. 
en dédoublant l'identité du père (Chouiten 154). Enfin, si ce prénom représente une identité du fils qui se définit par rapport à cet Autre si intime, le prénom Amer n'Amer tout en signifiant Amer fils d'Amer peut aussi être lu comme Amer fils de lii-même, c'est-à-dire littéralement "quelqu'un fils du même quelqu'un», conformément à ce que le personnage lui-même indique dans l'incipit de son journal. Car Amer a dû s'élever seul en l'absence de son père, être le rejeton de ses propres forces : «Il m'a fallu me débrouiller seul, tomber malade et attendre de guérir, porter ma faiblesse comme une exaspérante infirmité et attendre impatiemment d'être fort, ne pas bien comprendre nos mœurs, nos gens, notre morale, et grandir pour apprécier tout cela à sa juste valeur. » (CM 156)

Dans cette optique, tout en perpétuant le sang familial, Amer n'Amer représenterait également une cassure dans la lignée d'après l'allusion faite à l'absence du père ainsi que sa double origine de sang-mêlé.

Amer n'Amer peut être considéré comme son propre père ainsi que son propre fils, dans le sens qu'il doit forger son identité et réclamer sa place parmi les siens avec ses propres forces car cette identité ne va pas de soi pour un métis, contrairement au cas des "pur sang» (CM 124) du village. Son identité kabyle authentique lui est contestée par tout le village à cause de sa double origine et du fait qu'il est orphelin :

«Les gens délicats évitent de me faire sentir que je ne suis pas tout à fait de la famille. [...] La famille : je veux dire tous les autres. Ceux qui aiment affirmer lorsqu'ils parlent: 'Nous, Kabyles... nous, musulmans, nous, que Dieu n'a pas faits roumis...', les gens d'Ighil-Nezman, quoi. Les 'pur sang', qui se savent tels et font semblant d'oublier que je n'en suis pas un [...] » (CM 124).

$\mathrm{Au}$ lieu de se voir accorder automatiquement et avec bienveillance son statut d'enfant «de la famille », il doit le prouver et le réclamer: "Je lui ai tout de même prouvé que je suis un enfant d'Ighil-Nezman et non 'un bâtard étranger'. » (CM 132) Le prénom Amer n'Amer est en vérité le trophée d'une conquête identitaire.

«Avant de m'appeler Amer n'Amer, les enfants de mon âge m'appelaient 'fils de Madame', comme si je n'avais pas de nom. [...] tous s'acharnaient à oublier Amer : Amer mon père et Amer moi-même, comme s'ils voulaient effacer ce nom, nous l'enlever. » (CM 117)

À part «fils de Madame » et «bâtard étranger », il est aussi appelé « fils de mécréante » (CM 124-125) où l'allusion est encore faite à sa mère. Tous s'acharnent à insister sur sa différence due à son origine française maternelle au point de lui ôter son prénom kabyle, tout en substituant à ce dernier un surnom à la fois insultant ("fils de Madame», qui dans son cas sonne comme fils de sa mère) et insistant sur le côté roumi tout en effaçant sa part kabyle. Il lui faut proclamer le droit à un nom propre et local et, ce faisant, réhabiliter celui de son père défunt. Ce prénom se révèle ainsi être un cri revendicatif d'un métis qui opte pour son identité de Kabyle authentique et pour sa place dans la communauté à laquelle il estime 
appartenir, mais qui, pour sa part, hésite en revanche à lui reconnaître cette place légitime.

Qui plus est, ce choix semble suggérer la préférence d'Amer n'Amer pour son origine kabyle paternelle à l'origine française de sa mère ou encore à sa double origine. Non seulement prend-il les mesures nécessaires pour que les autres «disent entre eux Amer n'Amer et non 'fils de Madame' ( $C M$ 117), mais il affirme encore mieux aimer "être le fils de [son] père.» (CM 117) Quant à sa mère, elle entre aussi «dans l'ordre »: elle s'est kabylisée et est enterrée religieusement selon les coutumes kabyles. Elle « a fini dans la peau d'une croyante d'Ighil-Nezman. En un sens, c'est une bonne fin pour une Française devenue kabyle. » (CM 116) Tout doit, semble-t-il, converger vers la Kabylie qui se dresse comme le centre de la préoccupation et le lieu d'enracinement ultime d'Amer n'Amer, au point qu'il en devient valorisant pour une Française émigrante d'effacer son altérité pour devenir un Même. On constate encore ce fait dans la façon dont Amer n'Amer parle de lui-même. Il se qualifie à plusieurs reprises de «Bicot» $(C M 122,198)$ ou de « Noraf » $(C M$ 125, 126 , 200), sans tenir à mettre en avant sa semi-origine française. Ce sang-mêlé semble vouloir minimiser l'importance de la moitié française de sa double origine, et ce faisant il peut alors se déclarer Kabyle authentique. Contrairement aux écrits qui dénoncent le fait colonial et le regard orientaliste que le pouvoir hégémonique européen exerce sur les autochtones, ce roman place au centre le peuple autochtone minoritaire et les perspectives qui lui sont propres en renversant son rôle habituel d'Autre et son Altérité dans le discours colonial en celui de Soi autonomique, tandis qu'Autrui est représenté par les personnages encore plus marginalisés. L'homogénéisation s'opère dans une direction inverse dans ce roman : non celle des autochtones par les Européens, mais celle des individus qui s'apparentent à la culture occidentale par ceux qui réclament les traditions et les mœurs locales. L'identité et les traditions kabyles prévalent sur la francité et sur la mixité.

\section{Dehbia, la métisse culturelle}

L'hybridité de Dehbia, l'héroïne du roman, est le produit d'un mélange non biologique, ou, si l'on veut, celui d'un métissage socioculturel. Élevée dans un village kabyle de croyances chrétiennes, éduquée par des Pères et Sœurs Blancs, elle parle français et possède un nom de baptême. Après la mort de son père, sa mère Melha l'emmène du village chrétien d'Aït-Ouadhou pour Ighil-Nezman, son village natal de croyances musulmanes. Comme Amer n'Amer, Dehbia doit se confronter à l'hostilité de son village natal ainsi que de son village d'adoption, mais sans être un sang-mêlé comme son cousin, elle se voit tout autant rejetée à cause de son altérité par les habitants des villages de ses parents.

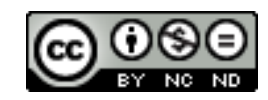


Sa différence tient d'abord à ses croyances chrétiennes au milieu des musulmans. Pratiquante, elle fréquente les religieux et l'église d'Ait-Ouadhou, suit les enseignements catholiques et sait réciter sa prière en kabyle et en français (CM 15). Elle se demande si la mort d'Amer n'est pas un châtiment de Dieu ou de Sainte-Marie (CM 35), et dans son désespoir, elle raisonne dans des termes pieux, prie et implore Dieu d'accepter Amer et de les réunir, elle et lui, dans son Royaume. Sa sincérité dans sa foi catholique contraste avec celle de beaucoup de chrétiens des Ait-Ouadhou qui, selon elle, "se sont convertis par intérêt » (CM 24) et «n'ont de chrétien que le nom» (CM 25). Ces simulateurs s'accommodent sans problème de leur catholicisme et leur origine musulmane. Lorsqu'un Père catholique voit un villageois converti faire ses prières à la mosquée, il s'en enquiert auprès de l'homme : "- Tu n'es pas musulman. - Pourquoi pas, mon père ? Je le suis de naissance. » (CM 26) Cette anecdote dépeint assez bien cet état de métissage culturel qui serait moins un état d'entre-deux que la manifestation d'un tiers espace si l'on suit la thèse de Nouss. Ce dernier distingue, d'un côté, l'entre-deux qui insiste sur la tension et la limite entre les deux forces se confrontant et, de l'autre côté, le tiers espace qui privilégie la rencontre des deux entités dans la proximité suite au déplacement hors de leurs propres frontières définies et qui «ignore» ou «occulte» la place de la frontière indispensable dans la définition de l'entre-deux. Le protagoniste de cette anecdote se situe dans une conjonction où les deux positions - être chrétien et musulman arrivent à coexister et cette proximité favorise ainsi la « juxtance nécessaire à la négociation » (Nouss 62) caractérisant le tiers espace. Debian, quant à elle, doit cependant dissimuler ses croyances et feint d'être musulmane lorsqu'elle arrive à Ighil-Nezman: "L'idée de vivre tout à fait en musulmane ne l'effrayait pas car, en dépit des apparences, elle avait assez de caractère et d'intelligence pour rester fidèle à son Dieu. » (CM 30) Or, malgré la différence entre les chrétiens $d u$ dimanche d'un côté et Dehbia, chrétienne sincère de l'autre, tous optent pour la même stratégie vis-à-vis de leur situation entre deux croyances et deux modes de vie : réconcilier l'apparence et la réalité tenue cachée afin de faciliter la vie au quotidien. Cette position flexible ferait sans doute de Dehbia une croyante, sinon hypocrite, du moins pragmatique, telle que ceux qu'elle dénigre, puisque, dans un sens, elle agit aussi "par intérêt» pour tenter de rendre invisible son altérité et atténuer son étrangeté. Ces personnes se trouvent dans un mi-lieu, c'est-à-dire un tiers espace où deux traditions se négocient. C'est un espace entre deux territorialités, mais qui n'est ni entièrement celui de l'un ni complètement celui de l'autre ; c'est à la fois la source et le produit des hybridations où des discours différents cohabitent et se confrontent en créant un territoire ambigu de conjonction. C'est ainsi que Dehbia finit par se préoccuper moins de sa foi que des choses futiles sur lesquelles l'attention des jeunes filles du village se focalise :

« [À] vivre dans ce milieu où la religion musulmane était réduite à quelques habitudes, quelques gestes sans portée et quelques superstitions sans conséquences, cela éteignit 
peu à peu son ardeur mystique ; bientôt elle se tourmenta moins pour l'Au-delà et s'intéressa davantage aux propos futiles, aux projets terre à terre, aux espoirs sans prétention de toutes ces filles pleines de santé et d'appétit. » (CM 31)

D'ailleurs, elle a déjà des manières de musulman lorsqu'elle vit encore au village chrétien : quand le Père Dubois lui répond avec justesse sans qu'elle pose ses questions, "elle [a] envie de courir lui embrasser la tête comme font les musulmans. » (CM 28) Les deux antipodes ne sont en réalité pas si indépendants l'un de l'autre.

La deuxième altérité de Dehbia qui incommode vient de son statut d'étrangère et de «bâtarde » $(C M 21,140)$. Sa mère Melha, en se mariant pour se débarrasser de sa réputation de fille volage («Chrétienne ou putain, il avait fallu choisir. » (CM 143)), part d'Ighil-Nezman pour Aït-Ouadhou, village natal de son mari où Dehbia a grandi. Cependant, les habitants d'Ait-Ouadhou traitent les deux femmes comme des étrangères parce qu'elles viennent d'ailleurs, d'un village musulman, et en raison du doute portant sur la parenté paternelle de Dehbia, au point qu'«il a fallu partir quand le père de Dehbia est mort. Personne quasiment n'a voulu [d'elles]. » (CM 140). Les habitants d'Ighil-Nezman ne veulent pas d'elles non plus car ces deux rapatriées représentent le déshonneur du village et parce qu'elles ont été ou sont encore chrétiennes. Bien que les chrétiens de Kabylie représentent seulement «une faible minorité [et soient] insignifiants, négligeables » (CM 23), le fait d'avoir fait partie de cette minorité n'est en rien insignifiant ni négligeable pour les gens d'Ighil-Nezman qui «[font] mine d'oublier » (CM 23) ce passé sans l'oublier nullement. La période de la vie dans le village chrétien reste gravée profondément dans l'image de ces deux femmes comme une marque ineffaçable de supplice. Être né au village ne signifie ni une réinsertion immanquable ni une appartenance spontanée. Le métissage de Dehbia est moins une question de kabylité comme c'est le cas pour Amer: il s'agit d'une hybridité socioculturelle à l'échelle de la double origine de deux villages kabyles et de deux mentalités qui fonctionnent comme deux mondes distincts. Son origine " étrangère " (CM 145), ses croyances religieuses ainsi que le fait d'être «bâtarde » sans soutien ni ressources - étant "fille infidèle sortie on ne sait d'où » (CM 42) - dans une société qui prône l'appartenance au réseau familial, font de Dehbia à la fois une Autre et une hybride à plusieurs égards. Elle incarne simultanément plusieurs territorialités à la marge de la société kabyle. Et malgré ses efforts pour se conformer aux normes locales, sa vie ne sera pas la même que celle des autres filles d'Ighil-Nezman. Personne ne pourrait vouloir de cette bâtarde. Le terme «bâtard» doit être entendu ici non seulement dans son sens propre signifiant une progéniture de deux « espèces » différentes ou un enfant hors mariage, mais aussi dans son sens figuré exprimant le côté grotesque et étrange de créature hors-norme et atypique : "C'est une étrangère, une chrétienne, une malheureuse. Ceux qui la dévorent des yeux quand elle passe voudraient bien la garder un jour, une 
nuit, mais pas tout le temps. » (CM 145) Mokrane, le personnage fanatique, pense même qu'elle mériterait d'être violée sans pitié et finit par le faire réellement. Il est impossible d'oublier sa différence dans ce village: «Personne n'oublie quoi que ce soit, Nana Melha avait du moins cette certitude : personne n'épouserait sa fille. » (CM 23) Bien qu'elle soit kabyle, elle ne sera pas acceptée parmi les « siens».

\section{Mokrane, le " pur-sang » : question de pureté du sang et de croyances}

Le personnage de Mokrane est à l'extrême opposé des personnages de l'entre-deux dans ce récit. L'essentialisme qu'il incarne contraste avec les différentes formes d'hybridité en créant une tension si forte que la collision qui en résulte aboutira à la fin tragique d'Amer et au désespoir de Dehbia. «Obéissant et fanatique à souhait»(CM 43), Mokrane représente les mœurs et les traditions d'une société figée dans les valeurs du passé, qui ne prend pas en compte la transformation fondamentale qui est en train de se produire ou qui s'en protège. Son passage en France n'est pas pour lui une initiation à l'ouverture sur un autre mode de vie : «la vie chez les infidèles ne lui a guère réussi. Sa place était par conséquent au village » (CM 43). La bâtardise d'Amer et de Dehbia offusque Mokrane, comme si son authenticité et le fondement de la société étaient menacés par leur présence. L'altérité que comporte nécessairement l'hybridité est perçue comme quelque chose de «monstrueux» et de «contre-nature». Elle est déstabilisante non seulement à cause de son caractère d'exception, mais aussi de sa «difformité » qui trahit une «transgression initiale 》 (Clavaron 55). Son fanatisme de croyant le conduit à voir en Dehbia une "petite chrétienne » et une « infidèle » «dont la beauté le nargu[e] et choqu[e] son âme de bon musulman fanatique » (CM 58), telle une présence diabolique à la fois irrésistible et destructrice : elle personnifie «ce rêve imprécis et irréalisable qui tourmente perpétuellement les insatisfaits, ceux qui recherchent l'impossible bonheur et finissent dans le désordre, l'irréligion et la honte» (CM 77). La violer serait même selon Mokrane un "acte pieux » qu'il se sent « en mesure d'accomplir sans pitié » (CM 58). L'ordre, la religion (du Même et non pas celle de l'Autre) et la dignité sont étroitement rattachés les uns aux autres pour édifier le centre des valeurs par rapport au désordre voire à la débauche de la marginalité et à la violation morale de ceux qui ne se conforment pas à ces règles. Ironiquement, Mokrane est lui-même la victime de l'autorité familiale prônant l'ordre comme valeur suprême. En acceptant le mariage imposé par sa famille pour les bénéfices qu'il apporte à la tribu, «il ne rest[e] plus à Mokrane qu'à être heureux parce que l'ordre, la religion, l'honneur, la famille [veulent] qu'il [soit] heureux »(CM 79). Mokrane représente un simple engrenage dans un appareil qui fonctionne en se conformant aux valeurs collectives d'une société kabyle figée dans le temps, tandis que les hybrides comme Amer ou Dehbia symbolisent déjà une société en 
transformation suite aux interactions de plus en plus fréquentes entre les cultures occidentales et kabyles.

\section{Mehla et Madame, exemples d'intégration}

Si Amer et Dehbia rencontrent tous les deux des situations d'entre-deux difficiles voire impossibles à surmonter, leurs mères ne semblent pas être tourmentées par ce dilemme. Elles s'adaptent volontiers. Marie et Melha ont toutes deux connu l'expatriation, se sont toutes les deux mariées à des étrangers, dans des circonstances différentes. Toutes deux s'efforcent de s'intégrer dans la communauté où elles ont élu domicile et elles ont toutes deux un(e) descendant(e) «bâtard(e)» (CM 21, 132, 140, 179, 207) qui a du mal à être pleinement accepté par les locaux. Néanmoins, Marie n'a pas choisi de rentrer dans son pays natal avec son enfant : elle choisit de rester en pays étranger pour y élever son fils, au pays de son mari ; elle veut même que son fils y reste sans qu'il aille en France comme c'est la coutume. Selon Amer : «elle n'avait qu'une idée : il fallait que j'aie une situation $[\ldots]$; un métier quelconque à exercer ici, et non que j'aie comme les autres à pérégriner en France.»(CM 179) Elle est décrite comme une femme aussi sage et avisée qu'une femme kabyle. D'ailleurs, malgré l'identité métisse de son fils, beaucoup de mères viennent lui proposer leur fille pour mariage. Quant à Melha, c'est une tout autre histoire. Bien qu'elle soit l'enfant du pays, elle ne trouve de refuge à son retour au village natal que chez cette Française, et personne ne veut prendre sa fille comme épouse ou bru. Ce décalage se constate dans les paroles de Melha à Marie :

"Tout le monde te respecte, toi. Tu es Française, tu n'as pas besoin d'eux, tu as un grand fils - que Dieu te le garde - ils te craignent et te respectent. Mais, moi ? Sais-tu ce que je vaux à leurs yeux ? [...] Ils se disent : «Une chrétienne, une femme sans honneur, qui a quitté le village on ne sait pourquoi, qui est revenue on se demande pour quelle raison... » (CM 46)

À vrai dire, personne ne connaît la véritable raison pour laquelle Marie a quitté son pays. Pourtant, cette Française immigrée est bien plus respectée que Melha, enfant authentique du village et rapatriée, mais méprisée et rejetée parce que l'on considère qu'elle déshonore «l'ordre» et le bon usage du lieu.

Pour ces deux mères qui portent en elles un itinéraire de deux pays, de deux cultures et de deux croyances, l'identité et la religion sont sujettes à négociation si l'on veut bien se faire accepter et reconnaitre. L'enracinement ou/et le rapatriement se réalisent seulement dans le conformisme aux conventions du lieu de l'insertion et dans l'effacement de la différence individuelle. Il s'agit de se fondre dans la foule et de cacher sa singularité. Outre la vie quotidienne, la religion est de même un terrain de négociation si l'on veut réussir son intégration. À ce propos, Melha est flexible: «Melha serait musulmane à Ighil-Nezman comme elle était 
chrétienne aux Aitt-Ouadhou » (CM 31), comme si la croyance n'était autre chose qu'une modalité de vie dont il fallait s'accommoder selon la situation dans laquelle on se trouve. Sa fille Dehbia reste ferme dans ses croyances, mais elle «ne t[ient] pas non plus à afficher ses croyances au milieu d'infidèles qui se moqueraient méchamment du miracle de l'incarnation et de tout ce qui, pour elle, était plus précieux que la vie : sa foi, son Dieu.» (CM 31) Toutes deux "évitent de se singulariser, elles pratiquent le ramadan et respectent [les] fêtes religieuses.» (CM 142) Ce jeu de cache-cache à lui seul donnerait droit à l'intégration au prix de sacrifier son identité première. Les caractéristiques de l'hybridité sont loin d'être des enjeux valorisants dans la société kabyle d'Algérie des années cinquante dépeinte dans ce récit. Qui plus est, on constate clairement une différence de mentalité sur ce sujet entre deux générations chez ces deux couples mère-enfant. Tandis qu'Amer et Dehbia souffrent de leur différence et doivent la dissimuler ou assumer le malheur s'ils la montrent ouvertement, leurs mères ne semblent pas être perturbées par l'obligation de se plier au monopole des traditions qui régit cette même communauté si oppressante pour les deux jeunes gens. Un changement est notable dans la mentalité de la société sous l'influence de plus en plus importante de l'Occident liée à l'ouverture inévitable de la société kabyle suite à l'émigration régulière des Kabyles vers la France ainsi qu'à l'éducation à l'école de la Troisième République.

\section{Les émigrants et l'enracinement ambivalent}

La société kabyle présentée dans Les Chemins qui montent, le deuxième volet de la « chronique allant de 1910-1950», se distingue nettement d'avec celle décrite dans son premier volet, La Terre et le sang, qui a pour décor la vie dans le même village de la génération précédente, pendant la période de 1910-1930. La Terre et le sang est un roman de rapatriement. Le village d'Ighil-Nezman en est le centre, la patrie ou la matrice qui nourrit, apaise, rappelle ses enfants égarés dans la terre d'exil au monde moderne et offre sans faute sa générosité de pays natal éternellement accueillant. L'image infernale des mines de France et celle du milieu ouvrier des quartiers du nord de Paris contrastent avec les villages haut perchés, les montagnes grandioses et une vie à la portée de l'homme modeste en Kabylie. Ce monde accepte et même absorbe la Française Marie comme l'une de ses enfants. C'est un monde stable et bienveillant dont les mœurs et les coutumes ne sont pas sans défaut, mais sont présentées d'une manière plutôt neutre. Or, Les Chemins qui montent raconte une tout autre histoire. A vingt ans et à une génération d'intervalle, le thème de cette deuxième partie de la saga vire au déracinement et à l'exil. La pratique de l'émigration devenant presque une norme, l'amertume qu'elle suscite chez la famille restée au village, si cette dernière se résigne pour la survie sans se plaindre dans La Terre et le sang, devient du ressentiment et du mépris envers ceux qui délaissent les enfants, les femmes et les vieux dans la misère et la faim 
pour poursuivre une vie d'aventure « au paradis des hommes». "Va-t'en! Partez tous, génération de lâches, ma malédiction vous accompagne!» (CM 187) vocifère une vieille au départ du bus rempli de nouveaux partants. L'univers est comme scindé en deux : d'une part, la Kabylie natale des traditions et de la misère, d'autre part la France terre d'exil, de la modernité et des aventures incertaines.

Ces deux mondes ne sont pas réconciliables pour la plupart des habitants d'Ighil-Nezman qui ne se soucient pas non plus de les réconcilier. La rencontre de deux peuples et de deux cultures due à ces «va-et-vient lugubres d'oiseau migrateur maudit» (CM 195) n'a pas porté de fruits de mélange, si bien que le couple mixte des parents d'Amer n'Amer et leur enfant métis présentent un cas unique qui accentue ainsi la cassure et l'instabilité que ces identités d'entre-deux représentent. Pour les autres, les deux mondes sont vécus si séparément que l'assimilation ne se réalise pas :

«Les garçons sont écartelés entre deux mondes différents et qui les sollicitent tour à tour sans pouvoir les retenir. Tant qu'ils vivent en France, les plus sérieux mènent l'existence régulière de l'ouvrier consciencieux : ils peuvent fréquenter une jeune fille, avoir une maitresse ou même se marier. Lorsqu'ils reviennent au pays, ils se replongent sans hésitation dans la vie du village et changent de peau pour la bonne raison qu'ils ont changé de climat. [...] Si, de nouveau, ils repartent en France, il faut bien qu'ils se réadaptent là-bas, en attendant de se réadapter ici, au retour. Tout se passe le plus naturellement du monde. » (CM 51)

Ils changent automatiquement de perspective quand ils changent de lieu. L'adaptation et la réadaptation se font avec naturel et flexibilité à cause de la nécessité, mais la terre de souche et celle de leur émigration sont écartées délibérément. La vie d'émigration en France n'effleure qu'à la surface ces enfants prodigues d'Ighil-Nezman, sachant qu'ils sont en terre d'étranger: «la vie moderne des capitales se déroule comme un film devant nos yeux indifférents. [...] Quand nous revenons chez nous, tout se passe comme si nous n'avions rien vu, comme si nous n'avions rien appris. » (CM 196) Le monde moderne qu'est la France n'entame l'univers archaïque d'Ighil-Nezman que dans le rare cas de mixité conjugale ainsi que sous la mission catholique dite civilisatrice. Quant à l'éducation à l'école de la Troisième République qui est une réalité non négligeable du processus de l'hybridation socioculturelle, Feraoun ne semble pas y porter beaucoup d'attention dans ce récit, lui pourtant si conscient de l'importance du sujet. L'altérité de la francité et la mixité biologique et culturelle sont ainsi accentuées comme des taches de couleurs à la fois vives et ambiguës, telles des intruses, dans un tableau où une couleur monochrome prédomine. Ainsi les deux mondes qui font également partie de la vie restent deux entités monolithiques dont l'influence réciproque reste minimale. Cela rejoint l'esprit pragmatique de négociation adopté par Melha à propos de la religion. Comme ces émigrants, elle s'adapte et se réadapte selon le lieu où elle se trouve pour des raisons pratiques, surtout dans une société où les traditions l'emportent sur l'excentricité. Mais le sentiment de flottement 
demeure chez les uns et les autres :

« $[\mathrm{O}] \mathrm{n}$ s'en va en France avec l'espoir de tout arranger, sans jamais parvenir à arranger quoi que ce soit. Pendant ce temps les hommes et les femmes vieillissent, les enfants poussent dans la misère, deviennent grands à leur tour et commencent à réfléchir. Un jour, ils se détachent des vieux, prennent en haine le pays et n'aspirent qu'à s'évader. Quand ils consentent à demeurer, ils savent que l'aventure les sollicitera tôt ou tard, qu'à son irrésistible appel il faudra bien qu'ils répondent [...]» (CM 53)

Le déracinement et l'abandon s'emparent ainsi de cette communauté comme un cercle vicieux décharnant et déstabilisant la collectivité encore soudée de La Terre et le sang et fait des Chemins qui montent un roman de révolte et de critique envers «la misère en Kabylie» dévastée par l'émigration version années cinquante.

\section{En guise de conclusion}

$\grave{A}$ travers les personnages principaux des Chemins qui montent se brossent les portraits de différents types d'entre-deux et d'hybridité. Écrit depuis l'intimité de cette société des subalternes de la colonisation, le roman aborde la place des différences identitaires des métis et des hybrides interculturels, qui sont davantage réduits au statut de subalterne au sein même de la collectivité colonisée, en leur donnant une voix et en les représentant, dans les deux sens du terme. L'analyse de ces figures hybrides épaule indubitablement l'idée selon laquelle l'hybridité est, comme Bhabha l'indique avec raison, ce lieu de négociation entre le Même et l'Autre, ce performatif qui imite le pédagogique tout en créant de nouvelles significations. Mais en même temps, l'hybridité s'apparente à la fois aux convergences et aux déchirements nés de la problématique du mélange ou encore de la réconciliation des deux cultures et deux identités qui se négocient ou s'entre-excluent dans ce milieu spatio-temporel spécifique qu'est la Kabylie - et l'Algérie à une échelle plus grande - de la première moitié des années cinquante.

Nombre des ouvrages postcoloniaux ou ceux en transition de l'ère coloniale vers l'ère postcoloniale mettent l'accent sur le monocentrisme et l'hégémonie du pouvoir colonisateur-dominant-assimilateur sous l'emprise desquels les identités et les cultures autochtones ainsi que l'hybridité transculturelle ou multiculturelle résultant de la mixité forcée ou volontaire se voient diminuées, dénigrées ou menacées. Dans ce roman il est tout aussi question de la confrontation et du rapport de forces entre une culture dominante, hégémonique et essentialiste et les identités périphériques des différences dans Les Chemins qui montent. Sa particularité réside dans le fait que l'idéologie dominante qui tient la place du centre par rapport aux différences identitaires qui se trouvent à la frontière liminale s'apparente non seulement à l'hégémonie imposée par le colonisateur français, mais aussi et grandement à l'essentialisme basé sur les traditions inébranlables et 
sur la pureté des races et des cultures. Les critiques de Feraoun, s'il y en a, ne se dirigent pas moins contre un fanatisme prônant une identité kabyle figée et intolérante à l'égard des hybrides qu'envers le racisme colonisateur. L'écrivain a pu constater avant l'heure ce qu'il y a de problématique dans cette mentalité monocentrique et, dans cette optique, son roman se dresse comme un avertissement. Vues une soixantaine d'années plus tard, on sait combien ces critiques s'avéreront être annonciatrices. 


\section{Bibliographie}

Bhabha, Homi. Les Lieux de la culture. Une théorie postcoloniale. Trad. Françoise Bouillot. Paris : Payot, 2007.

Chouiten, Lynda. "Hybridité et négociation culturelles dans La Terre et le sang et Les Chemins qui montent». Synergies Algérie 13 (2011) : 151- 161.

Clavaron, Yves. Poétique du roman postcolonial. Saint-Etienne : Publications de l’Université de Saint-Etienne, 2011.

Elbaz, Robert et Mathieu-Job, Martine. Mouloud Feraoun ou l'émergence d'une littérature. Paris : Karthala, 2001.

Feraoun, Mouloud. Le Fils du pauvre. Paris : Seuil, 1954 (1950).

---. La Terre et le sang. Paris : Seuil, 1953.

---. Les Chemins qui montent. Paris : Seuil, 1957.

---. Lettres à ses amis. Paris : Seuil, 1969.

Nouss, Alexis. Plaidoyer pour un monde métis. Paris : Textuel, 2005.

Simon, Sherry. Hybridité culturelle. Montréal : L'Île de la tortue, 1999. 